\title{
SELECTIVE REINFORCEMENT TO ENHANCE THE STRUCTURAL PERFORMANCE OF METALLIC COMPRESSION PANELS
}

\author{
Gary L. Farley* \\ Army Research Laboratory \\ Vehicle Technology Directorate \\ Langley Research Center \\ Hampton, VA 23681 \\ (757) 864-3091 \\ Gary.L.Farley@NASA.Gov
}

\begin{abstract}
$\underline{\text { ABSTRACT }}$
An experimental and analytical investigation of the influence of selective reinforcement on metallic panels with cutouts was conducted. Selective reinforcement was shown to be a weight effective concept for increasing structural performance of panels with cutouts designed to carry loads into the post-buckled regime. For instance, a selectively reinforced aluminum panel under shear load exhibited a 68 percent increase in specificbuckling load as compared to a geometrically comparable unreinforced aluminum panel. In comparison, a quasi-isotropic carbon-fiber-reinforced-polymer composite panel only produced a 45 percent higher specificbuckling load than the same unreinforced aluminum panel. Selective reinforcement offers the potential to tailor structural response through local strengthening and stiffening the structure for a broad range of structural application.
\end{abstract}

* Non-AIAA Member, Aerospace Engineer, Mechanics and Durability Branch. 


\section{INTRODUCTION}

Structural discontinuities can produce irregular load paths; local regions of high stress, excessive bending, reduced buckling load or even initiate failure. To compensate, a designer can thicken the structure, change the type of material used in the design or change the structural concept. Local thickening of the structure will increase structural weight and can cause assembly problems with adjacent components, potentially aggravating load paths or stress states. The use of alternate materials, such as different metallic alloys or changing from metallic to polymeric-composite materials, frequently incurs a cost penalty or creates a different set of problems / design issues in another region of the structure. Finally, significant departure from conventional design practice is frequently an option of last resort because of developmental risk and the potential for significant cost growth.

An alternative approach that results in little departure from convention is to locally modify the structure without altering the component thickness or increasing design complexity. In many structural applications the desired structural response can be achieved by selectively reinforcing a high stress or low stiffness region leaving the majority of the structure unaltered. Selective reinforcement, as presented in this paper, is the intelligent integration of a reinforcing material into a conventional material, such as aluminum. The depiction in Figure 1 shows a wing skin with cutouts and rows of fasteners that has been selectively reinforced. In this example, the reinforcement is integrated into the skin to enhance local stiffness, strengthen boltholes and reinforce the perimeter of a cutout.

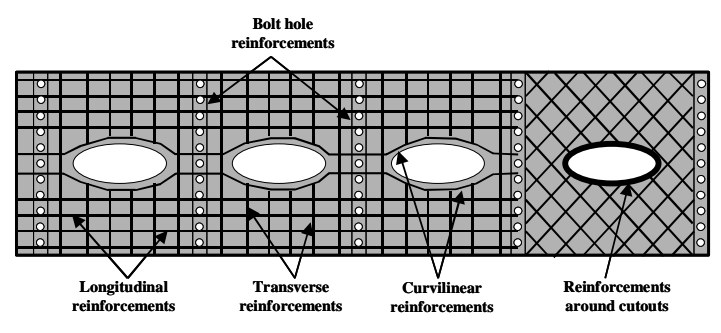

Figure 1. Depiction of a wing skin with multiple selective reinforcement architectures across the skin, around cutouts and along fastener rows.

Selective reinforcement of metallic automotive structures has been previously investigated. ${ }^{1,2,3}$ These studies concluded that a small percentage of reinforcement intelligently applied can dramatically influence structural performance. One such example is where ceramic fiber reinforced aluminum matrix inserts were cast into automotive brake calipers resulting in a stiffer, stronger and lighter weight structure. $^{3}$

Similar to structural fibers used in polymer matrix composites, the ceramic fiber used in selectively reinforced metals can be woven / braided or used in a unidirectional form. The aluminum matrix used to infiltrate the ceramic fibers is typically a near pure aluminum or aluminum with a small percentage of copper. At 50 percent fiber volume fraction the Young's modulus parallel to the fibers of the unidirectional ceramic fiber reinforced aluminum matrix composite material can be nearly 3.5 times that of unreinforced aluminum. At a 50 percent fiber volume fraction it has a 23 percent greater density than aluminum. Since it is used sparingly, any structural weight gain is small. When selective reinforcement is integrated into the structural design from the outset, a trade between structural performance and component weight can be made resulting in a lighter weight structure that has equal or superior structural performance than a comparable geometry unreinforced structure.

The objective of this paper is to present results of an investigation of square aluminum panels with cutouts that have selective reinforcement to enhance buckling and post-buckling response. The scope of this investigation includes testing of aluminum panels, with and without selective reinforcements that have circular cutouts and are subjected to inplane compressive load. Results from an analytical parametric study of panels having unidirectional $(0$ degree) reinforcements and bi-directional (0/90 and \pm 45 degree) reinforcements are also presented. Loading conditions used in the parametric study are inplane axial compression, equal magnitude bi-axial compression and shear. Results of this parametric study are compared with results from panels that are geometrically comparable, however composed of unreinforced aluminum or quasi-isotropic carbonfiber-reinforced-polymer composite material. A discussion of how to tailor structural response using selective reinforcement is also presented.

\section{EXPERIMENT}

This section provides a description of both unreinforced and reinforced test specimens and how the specimens were fabricated. A description of the 
test apparatus, test machine and test condition is also presented.

\section{Specimen description}

Both unreinforced and reinforced test specimens are flat panels 10.0 " wide by 10.0 " high and 0.1 " thick. A circular cutout was machined into the geometric center of the specimen. Cutout diameters were 3.0" and 5.0" resulting in diameter (D) to width $(\mathrm{W})$ ratios (D/W) of 0.3 and 0.5 , respectively. All specimens were fabricated from 7075-alloy aluminum. The opposite ends of panels were machined flat and parallel, and perpendicular to adjacent edges.

Reinforced test specimens were produced by machining grooves into the front and back surfaces of unreinforced aluminum panels in a back-to-back arrangement. Unidirectional reinforcements were soldered into the grooves. Groove width was approximately 0.5 " and 0.020 " deep. The most interior grooves were machined tangent to the cutout. The distance between grooves are 0.75 " and 0.25 " for panels with 3.0" and 5.0" cutouts, respectively. Groove depth at reinforcement intersection for bidirectional reinforced panels was approximately 0.040" deep. Groove depth for bi-directional reinforced panels increased from 0.020 " to 0.040 " in a linear manner starting approximately 0.5 " from the reinforcement intersection. A picture of a 0 degree reinforced panel is shown in Figure 2.

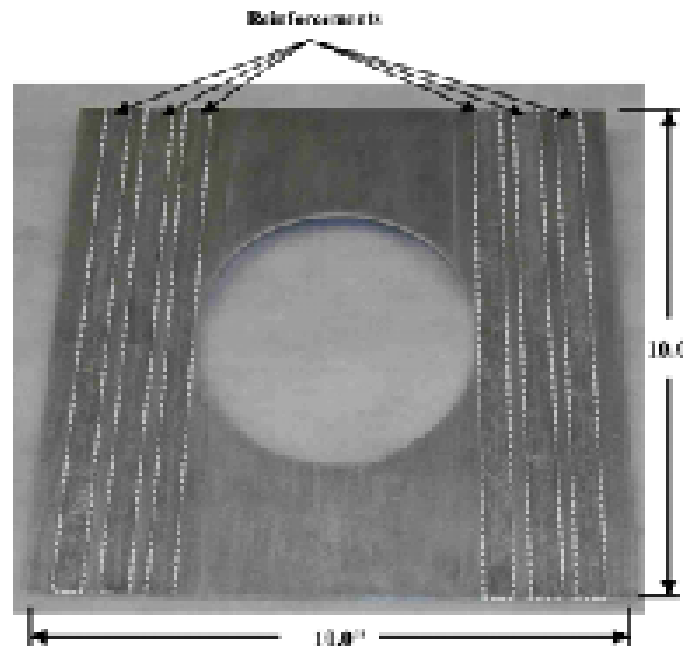

Figure 2. Unidirectional (0 degree) reinforced panel with 3.0 " cutout.

The unidirectional reinforcement is a continuous alumina oxide $\left(\mathrm{Al}_{2} \mathrm{O}_{3}\right)$ fiber inserted in a pure aluminum matrix. Fiber volume fraction was approximately 43 percent and the part was slightly narrower than the groove width. Reinforcement thickness was approximately 0.017 inches. A solder interface thickness of approximately 0.003 in. was sought to create a smooth panel exterior.

\section{Test apparatus and test conditions}

Multiple resistant strain gages were mounted on the surface of each specimen to provide strain measurement parallel to the direction of the applied load. Two linear variable displacement transducers were mounted normal to the panel to measure out-ofplane displacement. These transducers were located at the top and side of the cutout along the panel's longitudinal and transverse axis adjacent to the edge of the cutout. Displacement transducers were also mounted to the test machine's load platen to measure load platen displacement during the test.

Supports were mounted to each specimen to constrain out-of-plane displacement without restraining rotation along the unloaded edges of the specimen. Load introduction plates were clamped to the top and bottom edges of the panels. These plates facilitate uniform in-plane displacement along these edges and constrain specimen out-of-plane displacement along the loaded edges of the panel. The test machine load platen was leveled relative to the load introduction plates to minimize the introduction of bending into the panel. Load was applied to the specimen at approximately $1000 \mathrm{lb}_{\mathrm{f}}$ per minute. Load, strain and displacement data was recorded using an automated data acquisition system at a $1 \mathrm{~Hz}$ rate.

\section{ANALYSIS}

A parametric study was performed using finite element models, similar to the model depicted in Figure 3, to investigate the potential performance gain achievable by selectively reinforced panels. The parameters chosen for this study were based on preliminary results using quarter symmetric panels with cutouts. Various linear, curvilinear and sheet reinforcement architectures were investigated and based upon those results, it was concluded that this study should focus on unidirectional ( 0 degree) and bi-directional ( $0 / 90$ and \pm 45 degree $)$ straight reinforcements. All panels analyzed in this parametric study were 0.10 " thick and had the same material mechanical properties as the laboratory test panels. The effect of cutout diameter was investigated in the preliminary investigation and the trends relative to the effects of reinforcement architectures were independent of cutout diameter 
size so this parametric study is limited to panels with 3.0" diameter cutouts. Panels were subjected to inplane axial compression, bi-axial compression and shear loading conditions. The magnitude of the biaxial compression was equal in both directions.

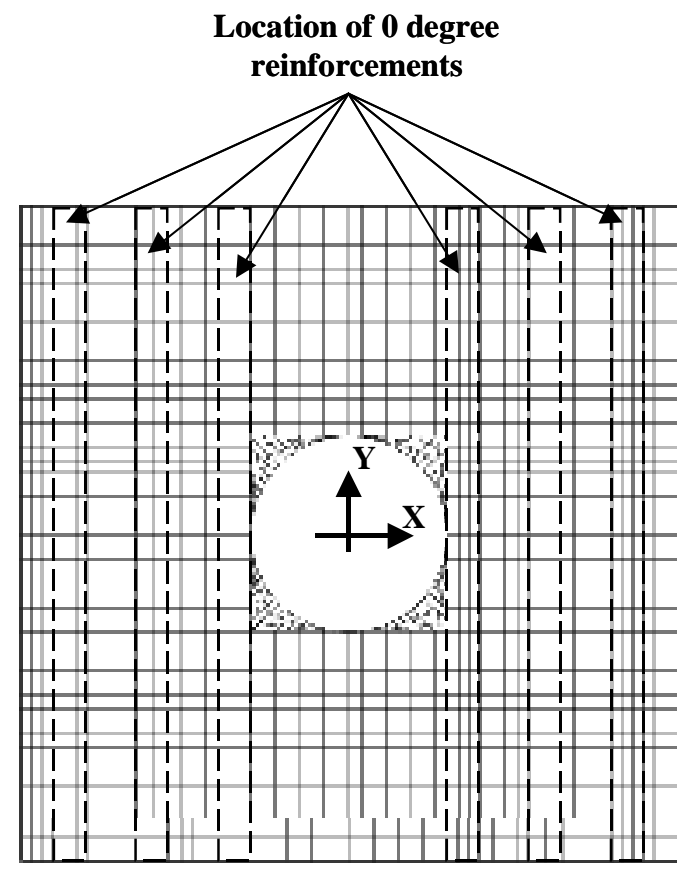

Figure 3. Finite element model of panel with 3.0 " cutout.

The boundary conditions for the axial compression case were similar to the boundary conditions created by the test fixture. Out-of-plane displacements around the perimeter of the panel were constrained at approximately 0.25 " from each edge. A uniform axial displacement was applied along the top edge of the panel to compress the panel. The displacements along the bottom edge of the panel were constrained in the axial direction. The applied load was calculated from the reaction forces along the bottom edge of the panel. The boundary conditions for the bi-axial compression case was similar to the axial load case except a uniform transverse displacement was applied to one side edge of the panel and the corresponding displacements at the opposite side were constrained.

For the shear load case the out of plane displacements were constrained around the perimeter of the model. A uniform horizontal displacement was applied along the top of the panel and a constant vertical displacement was applied based upon the desired shear strain. All inplane displacements were constrained along the bottom edge. A linearly varying transverse and axial displacement was applied to both panel's vertical edges. The resulting applied shear force was calculated from the reaction forces along the bottom edge.

Individual finite element models were created for panels with different reinforcement architecture. The models were developed in such a manner as to discretely model the reinforcements. The same finite element model was used for reinforced and unreinforced panels by adjusting the material stiffness of elements where the reinforcements reside. All analyses were material linear elastic. Geometric linear, geometric nonlinear and a buckling finite element analysis were conducted using the ABAQUS finite element computer program on all panel configurations in combination with all loading cases. ${ }^{4}$ Six node triangular and eight node quadrilateral finite elements were used that had membrane and bending stiffness.

The response of the reinforced panels is compared to the response of a geometrically comparable unreinforced aluminum panel. Also for comparative purposes the response of a carbon-fiber-reinforcedpolymer-matrix composite panel is included. The polymer-composite panel has a quasi-isotropic $(0 / 90 / \pm 45)_{s}$ lay-up and has a similar total thickness as the metal panels. The material properties used for modeling the unreinforced, reinforced and polymercomposite panel is included in Table 1.

\begin{tabular}{|c|c|c|c|c|}
\hline Material & $\begin{array}{c}\mathbf{E}_{11} \\
(\mathbf{M s i})\end{array}$ & $\begin{array}{c}\mathbf{E}_{22} \\
(\mathbf{M s i})\end{array}$ & $\begin{array}{c}\mathbf{G}_{12} \\
(\mathrm{Msi})\end{array}$ & $\mathbf{\prime}_{12}$ \\
\hline $\begin{array}{c}7075 \\
\text { Aluminum }\end{array}$ & 10.0 & 10.0 & 3.84 & 0.3 \\
\hline $\begin{array}{c}\text { Alumina oxide } \\
\text { reinforced } \\
\text { aluminum }\end{array}$ & 35.0 & 19.0 & 6.72 & 0.25 \\
\hline $\begin{array}{c}\text { Gr-Ep } \\
\text { composite }\end{array}$ & 20.0 & 1.3 & 0.62 & 0.32 \\
\hline
\end{tabular}

Table 1. Material properties used in finite element analyses.

A metric in terms of a normalized specific-buckling load for all panels was used. Specific-buckling load is a panel's buckling load divided by the average panel density. The normalized specific-buckling load metric is the quotient of the specific-buckling load of the reinforced panel and the specific-buckling load of the unreinforced panel; minus one. The resulting metric is a percentage change in performance relative 
to the unreinforced panel. Any positive value represents an improvement in specific-structural performance. The buckling response of the composite panel is also compared to the metallic unreinforced panel in the same manner. Postbuckling slope is presented using a similar normalized metric as the buckling-load metric.

\section{$\underline{\text { RESULTS }}$}

This section contains a discussion of the experiment results associated with the test specimens and the analytical results from the parametric study. A discussion of tailoring panel response using selective reinforcement is also included.

\section{Experiment}

The load-shortening response for the unreinforced and unidirectional ( 0 degree) reinforced test specimen with a 5.0" diameter cutout is depicted in Figure 4. Both panels exhibit bi-linear pre-buckling and post-buckling responses, excluding the panel's response at low applied loads. The nonlinearity in the pre-buckling response for both panels is attributed to panel imperfections and uncontrolled tilting of the test machine load platen during the test. The reinforced panel exhibited a 33 percent higher buckling load than the unreinforced panel.

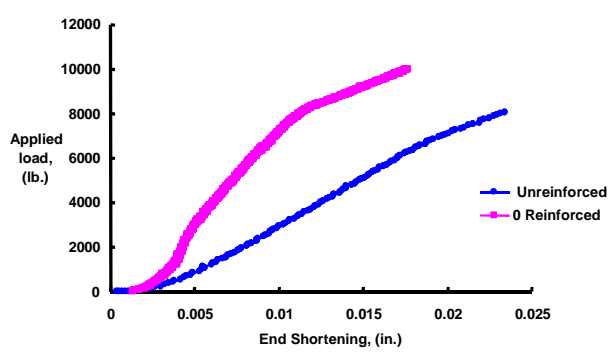

Figure 4. Load-shortening response of panel with 5.0" cutout

The load-shortening response for the unreinforced and bi-directional reinforced (0/90 degree) test specimen with a 3.0" diameter cutout is depicted in Figure 5. The unreinforced panel exhibited the anticipated near bi-linear pre-buckling and postbuckling response, excluding the response at low loads. The 0/90-reinforced specimen had two reinforcements delaminate from the panel that precipitated local panel buckling. The reinforcements continued to delaminate with increasing out-of-plane displacement and the test was halted. The load at which delamination occurred was 18 percent higher than the measured buckling load of the unreinforced panel. The reinforcements delaminated along their solder joints. Both reinforcements were adjacent to the cutout, one oriented longitudinal to the applied load direction and one transverse. Based upon a post-test visual inspection of the failed reinforced panel it was determined that delamination was caused by a weak solder joint produced by an insufficient amount of solder in the joint.

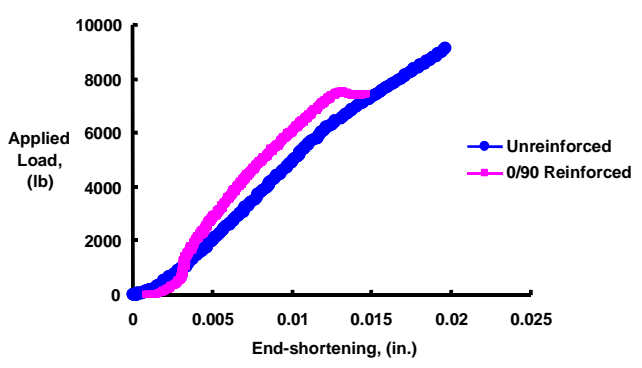

Figure 5. Load-end shortening response of panel with 3.0" cutout

Based upon these tests it was clear that the reinforcement significantly increased the buckling load of these panels. These improvements were achieved even though panel imperfections, load platen tilting and reinforcements delaminated on one panel.

\section{Parametric study}

A comparison of the general response of unreinforced and reinforced panels is initially presented. In subsequent sections a comparison of the panel's buckling load and post-buckling slope is presented for each load condition (axial, bi-axial, and shear). Finally, a discussion of tailoring panel response using selective reinforcement is presented.

\section{$\underline{\text { General response }}$}

An important aspect of any new structural concept is whether its response is similar to conventional structures. First, it is important to know whether the reinforced panels exhibit similar overall response as the unreinforced panels. Geometric nonlinear analyses were conducted for all panel combinations of configuration and loading conditions. All panels exhibited the characteristic bi-linear load-shortening 
response. Panel responses for bi-axial compression load condition are shown in Figure 6.

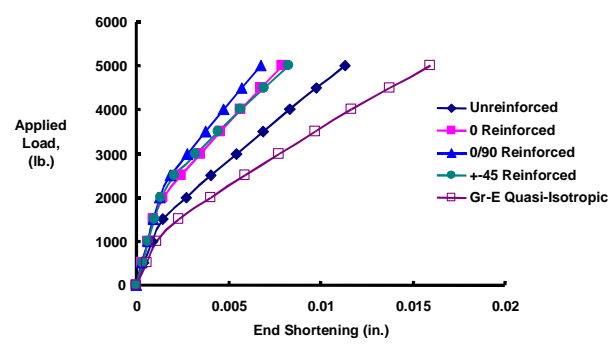

Figure 6. Load-shortening curve for all panel configurations subject to bi-axial compression load.

Prior to buckling, for both unreinforced and reinforced panels, the high stress region is adjacent to the cutout, as depicted in Figure 7. In the reinforced panel, load was primarily carried by reinforcements adjacent to the cutout and the magnitude of load diminished in the out board reinforcements. In the post-buckled state, the load shifted outboard to the panels edges, as shown in Figure 8. The reinforcements remained the primary load-carrying element in the reinforced panels. The stresses in the unreinforced part of the panel were generally much lower than the stresses in the reinforcement. Finally, the buckle mode shapes for both reinforced and unreinforced panels are almost identical, as demonstrated by the first modes in Figure 9. All panel configurations and combination of loads exhibited these pre- and post-buckling load and buckling mode characteristics.
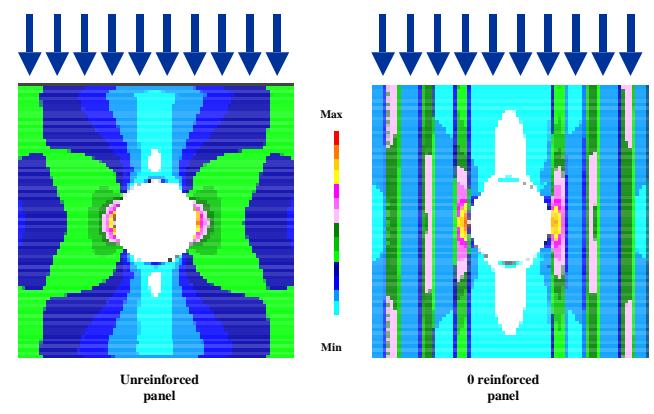

Figure 7. Von Mises stress contours of unreinforced and 0 degree reinforced panels prior to buckling. (axial compression load)

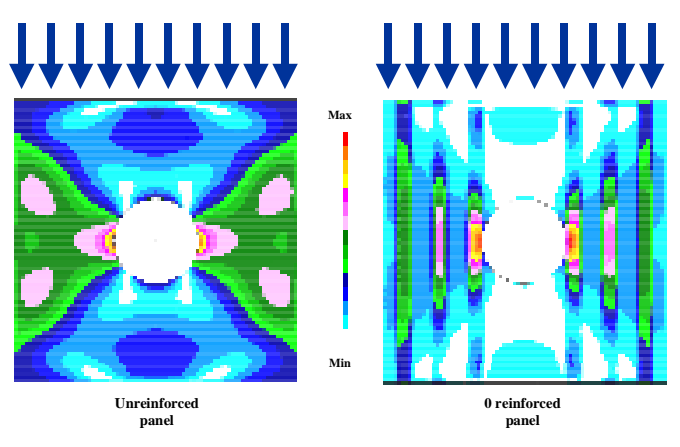

Figure 8. Von Mises stress contours of unreinforced and 0 degree reinforced panels post buckled. (axial compression load)
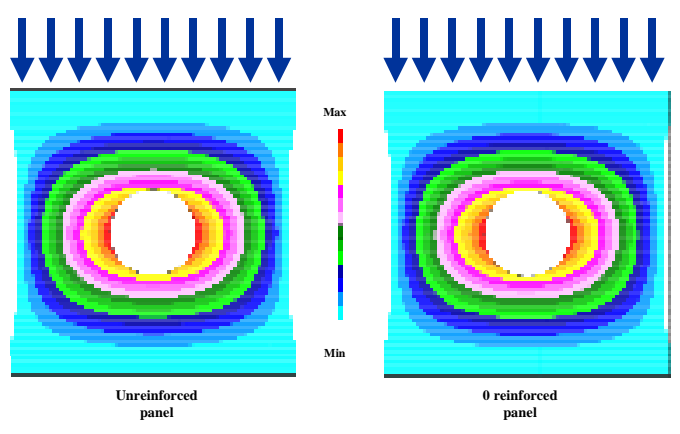

Figure 9. First buckling mode shape of unreinforced and 0 degree reinforced panels.

(axial compression load)

\section{$\underline{\text { Axial loading condition }}$}

The normalized specific buckling loads for the reinforced and polymer composite panel for the axial load condition are presented in Figure 10. The 0 degree reinforced panel exhibits approximately a 28 percent increase in the normalized specific-buckling load. Both bi-directional reinforced panels, $(0 / 90)$ and $( \pm 45)$, exhibit 46 and 47 percent increase, respectively, in normalized specific-buckling load, whereas the polymer composite panel exhibited only a 30 percent increase. The difference in performance of the 0 degree and the both bi-directional reinforced panels is attributed to the lateral stiffening effect of the 90 or 45 degree reinforcements in the bidirectional reinforced panels.

The buckling load of the reinforced panels was higher than the unreinforced panel whereas the buckling load of the polymer composite panel was lower than the unreinforced panel. Also, the weight of the reinforced panels was slightly greater $(2-5$ percent) than the unreinforced panel whereas the 
weight of the polymer composite panel was substantially less, approximately 40 percent less).

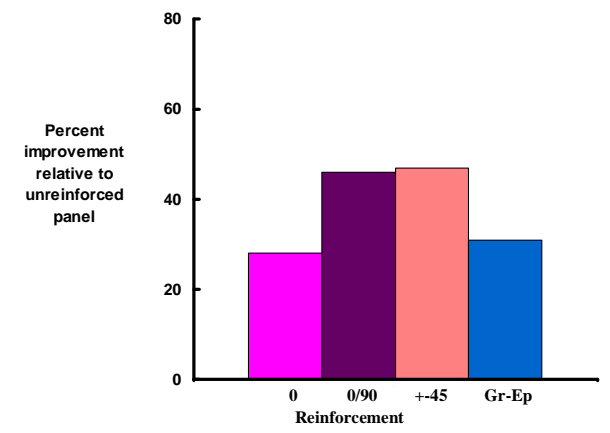

Figure 10. Percent improvement in normalized-buckling load of panels subjected to axial compression load.

The selectively reinforced panels exhibited improvement in normalized specific post-buckling slope for axial loading condition, as shown in Figure 11. A 29, 34 and 15 percent higher value of normalized specific post-buckling slope was obtained for $0,0 / 90$ and \pm 45 degree reinforced panels, respectively, than the unreinforced panel. The polymer composite panel produced a 17 percent increase in normalized specific post-buckling slope.

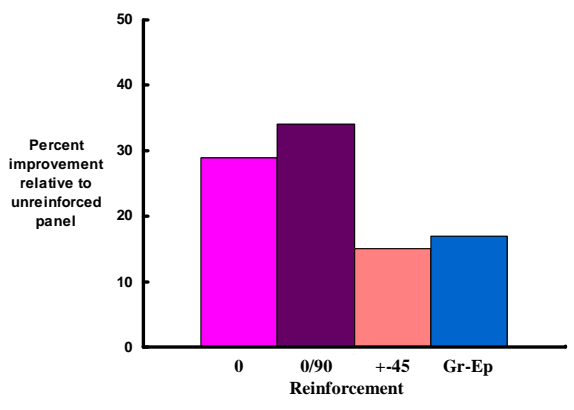

Figure 11. Percent improvement in normalized specific post-buckle slope of panels subjected to axial compression load.

The 0 and 0/90 degree reinforced panels exhibited the highest increases because their reinforcements extended the length of the panel and some reinforcements were adjacent to the supported sides where the load shifted after the panels buckled. The 90-degree reinforcements in the 0/90 degree reinforced panels provide additional panel stiffening in the post-buckling region in a similar manner as it provides to increase the buckling load. Thus, the higher value for the $0 / 90$ degree reinforced panel than produced by the 0 degree reinforced panel.

\section{$\underline{\text { Bi-axial loading condition }}$}

The normalized specific-buckling response for reinforced and polymer composite panels subjected to bi-axial loading are presented in Figure 12 . The 0 degree reinforced panel exhibited a 26 percent increase in normalized specific-buckling response, whereas the $0 / 90$ degree and \pm 45 degree reinforced panels exhibited a 46 and 47 percent increase, respectively. The polymer composite panel exhibited a 42 percent increase, a significant improvement relative to the axial load condition. The 0 degree reinforced panel exhibited a 2 percent decrease in performance relative to the axial load case. The performance decrease of the 0 degree reinforced panel is due to the lack of reinforcement oriented in the transverse direction to react the transverse component of the bi-axial load. Both of the bidirectional reinforced panels exhibited performance levels similar to those achieved from the axial load condition, which is attributed to having equal amounts of reinforcement in both directions. The polymer composite panel's performance significantly improved relative to the axial loading case. In this loading situation the \pm 45 and 90 degree reinforcements of the composite panel are contributing to the reaction of the transverse loads.

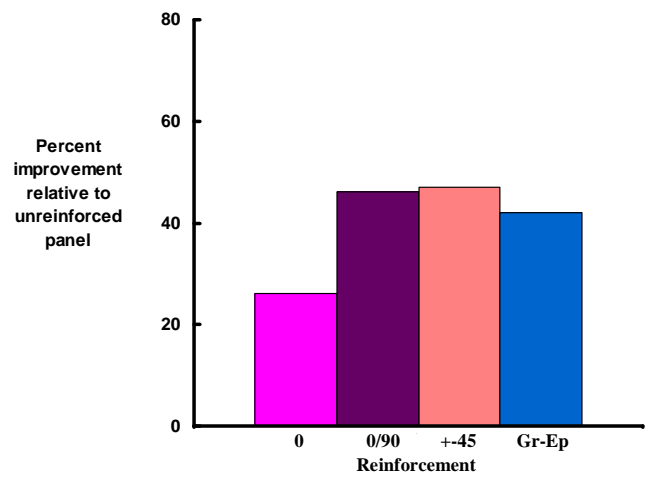

Figure 12. Percent improvement in normalizedbuckling load of panels subjected to equal magnitude bi-axial compression load.

Similar to that exhibited by the axial load case, all of the selectively reinforced panels had greater buckling loads and greater panel weight than the unreinforced panels. Thus, the performance gain by the reinforced panels is due to improved structural performance. However, the buckling load of the composite panel is 
less than the buckling load of the unreinforced panel and the high performance metric of the composite panel is attributed to the lower weight of the composite panel.

The specific post-buckling slope performance for the selectively reinforced panels under bi-axial load, as depicted in Figure 13, is similar to the results obtain under axial load. That is, the 0/90 degree reinforced panel had the highest improvement in normalized specific post-buckling slope, 31 percent, relative to the unreinforced panel and the \pm 45 had the least improvement, 12 percent. The polymer composite panel exhibited a 21 percent improvement. Similar to the axial load case, reinforcements parallel to the direction of the applied load and near the panel's edges have the most influence on post-buckling slope.

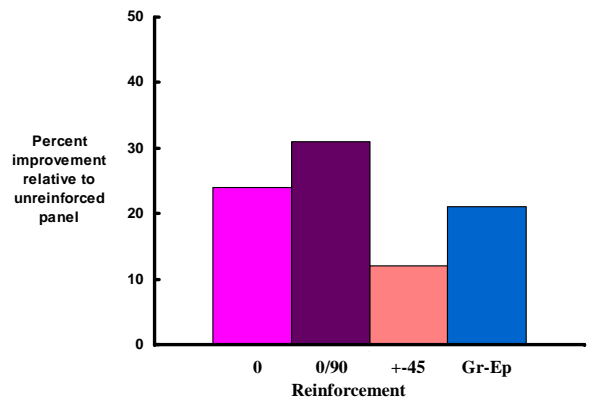

Figure 13. Percent improvement in normalized specific post-buckle slope of panels subjected to equal magnitude bi-axial compression load.

\section{Shear loading condition}

The normalized specific-buckling response for these panels subjected to shear loading is presented in Figure 14. The 0 degree and 0/90 degree reinforced panel exhibited a 24 and 36 percent, respectively, increase in normalized specific-buckling response. The \pm 45 degree reinforced panel exhibited a 68 percent increase in specific-buckling response. This dramatic increase was directly related to the significant increase in shear stiffness provided by orienting the reinforcements at parallel to the local principal load direction $( \pm 45$ degrees). The polymer composite panel exhibited a 45 percent increase in normalized specific-buckling response.

Similar to that exhibited by the axial and bi-axial load case, all of the selectively reinforced panels had

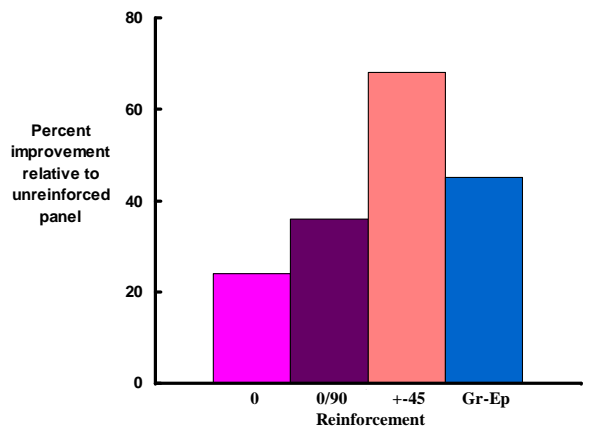

Figure 14. Percent improvement in normalized-buckling load of panels subjected to shear load.

greater buckling loads and greater panel weight than the unreinforced panel. Therefore, performance gain by the reinforced panels was due to improved structural performance. Also, the buckling load of the composite panel is less than the buckling load of the unreinforced panel and the high performance metric of the composite panel is attributed to the lower weight of the composite panel.

The 0 and 0/90 degree reinforced panels exhibited the lowest improvement in normalized specific postbuckling slope of 3 and 10 percent, respectively, as shown in Figure 15. The unidirectional

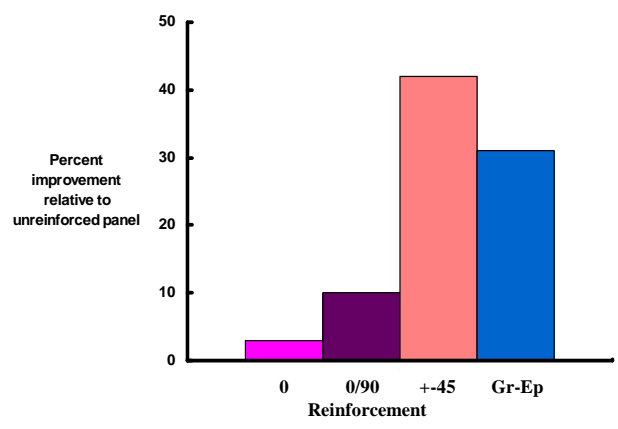

Figure 15. Percent improvement in normalized specific post-buckle slope of panels subjected to shear load.

reinforcement in the 0 and $0 / 90$ reinforced panels are not oriented in the principal load directions $( \pm 45$ degree) so their contribution to shear stiffness is less than if they were oriented in the \pm 45 degree direction. The \pm 45 -reinforced panel produced the highest improvement in normalized specific post-buckling slope, 42 percent, because its reinforcements are oriented in the principal load direction. The polymer 
composite panel produced a 31 percent improvement due to their \pm 45 layers.

\section{$\underline{\text { Structural tailoring with selective }}$ $\underline{\text { reinforcement }}$}

This investigation has shown selective reinforcement to be an effective concept to enhance structural performance through tailoring local material strength, pre-buckling stiffness, buckling load, and postbuckling stiffness. To exploit the tailorability of selective reinforcement it is necessary to understand the structural response mechanisms, when each response mechanism becomes dominant, any interaction between mechanisms and the mechanical properties and applicability of the reinforcing materials.

Structural tailoring becomes increasingly complicated the more design constraints that are imposed. For instance, consider two panels that are each optimally designed to carry the same load into the post-buckling regime. One panel has an additional requirement of a specified buckling load. Since it is possible to tailor the pre-buckling stiffness, buckling load and post-buckling stiffness to achieve the optimal reinforcement architecture, the resulting panel stiffness and the weight of these panels will differ. Although some "rules of thumb" can be established for selectively reinforced structures, the optimal design can best be achieved using computer based automated design tools due to the multiplicity of design variables and constraints.

Based upon the results from this study the following "rules of thumb" seem applicable:

1. To increase the buckling load it is necessary to first determine the location on the panel that will buckle. Reinforcement should be applied at the surface of the panel at the anticipated buckle site to maximize bending stiffness. The reinforcement fibers should be oriented parallel to the panel's principal load direction. To locally increase-bending stiffness using narrow reinforcements it is more efficient to increase reinforcement width than reinforcement thickness.

2. To increase a panel's post-buckling slope, reinforcements should be applied along the edge of the panel with the unidirectional reinforcement fibers parallel to the local principal load direction. Reinforcement should be applied near the panel's surface to maximize bending stiffness. To locally increase bending stiffness using narrow reinforcements it is more efficient to increase reinforcement width than reinforcement thickness.

3. Shear stiffness can be significantly improved through the orientation of reinforcements at \pm 45 degrees.

4. Bi-axial stiffness can be effectively achieved using either $0 / 90$ or \pm 45 reinforcements.

\section{CONCLUSIONS}

An experimental and parametric analytical investigation of the influence of selective reinforcement on metallic compression panels with cutouts has been conducted. It has been shown that the selectively reinforced panels exhibit similar global responses, such as mode shapes and load shift after panel buckles, as that of unreinforced metallic panels.

Selective reinforcement, as applied in this study, results in a small weight gain, approximately 2 to 5 percent, depending upon the reinforcement architecture. Even though there was a weight gain, the specific buckling response, which includes the relative weight of the panels, exhibited between 23 to 68 percent increase relative to unreinforced aluminum panels. In the limited cases shown in this study, the selectively reinforced metal panels exhibited up to a 15 percent higher performance than the geometrically comparable fiber-reinforced polymer composite panels even though the average density of the selectively reinforced panel was over 60 percent higher than the polymer composite panel.

This dramatic performance increase shows the potential of this design concept for metallic structures. If reinforcements were integrated into the original structural design it would be possible to trade increased performance for reduced structural weight, that is create a higher performing structure that weighs less than a conventional metallic structure.

\section{REFERENCES}

1. Degischer, H.P., Rammerstorfer, F. G. and Beffort, O., "Design Rules for Selective Reinforcement of Mg-Castings by MMC Inserts," Magnesium Alloys and Their Applications, Wiley-VCH, pp. 207-214, 2000.

2. W.H. Hunt, Jr., and D.B. Miracle, Automotive Applications of Metal-Matrix Composites, Composites, Volume 21, ASM 
Handbook, Edited by D.B. Miracle and S.L.

Donaldson, pp 1029-1032 @ 2001 ASM

International.

3. Anon., "Aluminum Matrix Composites (AMC) Reinforced Brake Calipers," 3M

Corporation, Product literature, 1998.

4. Anon., ABAQUS users manual, Hibbitt, Karlsson and Sorensen, Inc., 2000. 